SLAC-PUB-8061

February 1999

\title{
A Novel Wire Scanner for High Intensity Pulsed Beams
}

\author{
C.H. Back ${ }^{\dagger}$, F. King, G. Collet, R. Kirby and C. Field \\ Stanford Linear Accelerator Center, Stanford University, Stanford, CA 94309, U.S.A.
}

\begin{abstract}
A novel wire scanner has been developed with applications at linear accelerators where small beam spots or high intensities would otherwise destroy wires. A pattern of wires is supported on a thin membrane and remains in place even when punctured by the beam. Many scans can be made before the material needs to be replaced. Tests of the principle have been carried out at the Final Focus Test Beam at SLAC.
\end{abstract}

Submitted to Nuclear Instruments and Methods in Physics Research

Work supported by US Department of Energy, Contract DE-AC03-76SF00515

$\dagger$ Present address: FKP-Mikrostrukturforschung, ETH-Hönggerberg - HPT C2.2, CH-8093 Zürich. 


\section{Introduction}

Wire scanners are an important diagnostic tool for high energy particle beams. Various types have been developed for use with the pulsed beam at the SLC [1-5]. Because of the effects of beam induced heating, even fine carbon wires are limited to pulse intensities $<10^{10}$ electrons for spot sizes given by $\sigma_{x} \times \sigma_{y}<3 \mu \mathrm{m}^{2}$ [5]. In this paper we describe an attempt to use fine wires supported on thin membranes as a way to allow even damaged wires to be used many times. Examples were tested at the Final Focus Test Beam (FFTB) at SLAC.

The FFTB is an external electron beam line from the SLAC linac. It is designed to make use of the low emittance delivered by the damping rings and hardly diluted by the linac. The linac beam is directed into a five-stage beam line where a demagnification of more than 300 leads to spot sizes as small as $\sigma_{\mathrm{y}}=70 \mathrm{~nm}$ (vertical plane) by $\sigma_{\mathrm{x}}=1 \mu \mathrm{m}$. At normal operating intensities $\sim 10^{10}$ per bunch, this immediately destroys scanning wires. Other techniques, slower, less flexible and expensive, must be used to measure the spot size [6].

A substantial effort is required to tune the beamline, and it would benefit from a fast wire scanner with the ability to measure much smaller spot sizes than is now possible. In pursuit of this goal, the apparatus described here was designed to maintain the compact dimensions of the carbon-wire scanner which it replaced, while staying compliant with the standard SLC wire scanner control package.

\section{Principle of the Technique}

In a regular scanner, wires are stretched between prongs of a rigid "fork". During a scan, the position of the wire is moved in small uniform steps, pulse by pulse, relative to the center of the beam. The overlap between wire and beam is measured, typically using the Bremsstrahlung signal. If the energy deposited in the wire is enough to break it, the ends separate and the wire is effectively lost. As a way of preventing this, we have attempted to substitute a grid of metal lines prepared lithographically on a thin substrate. If the strongly focussed beam hits a metal line it will produce a Bremsstrahlung signal similar to that of a carbon fiber, and so can be used to probe the size of the beam. If the metal line is damaged by the beam, however, the damage is local, and the rest of the line, and all the other lines, remain intact. To continue the scan, it is only necessary to target the beam at another line, with an offset relative to its center corresponding to the next point in the scan. This is illustrated in Fig. 1. In relevant cases, in fact, damage will occur with every beam pulse.

The scan may proceed by stepping either perpendicular to the metal lines (Figure 1a) or along a line (Figure 1b). In the former case the step size is the line-to-line spacing plus the scan-step change in the relative offset between the beam and the wire. If, for example, the lines are 20 microns apart and it is desired to sample the beam-wire overlap at 0.1 micron steps, then the linear stage moves in 20.1 micron steps between beam pulses. At FFTB the default scan uses 100 of these samples, or 99 steps, and so would be $1.99 \mathrm{~mm}$ long. The stage motion must be linear over this range, while maintaining 0.1 micron resolution.

The case where the principal scan-step is parallel to the metal line requires steps long enough to be clear of the damage crater from the previous shot. Experience shows that this 
can be 15 microns wide. At the same time as the long step parallel to the line, a scan-offset step must be made perpendicular to it, in order to scan the wire across the beam. Both motions may be made with the mechanical scanner, or the finer one may be carried out by synchronously deflecting the beam with electromagnets. In this case, the major motion must be aligned accurately with the lines (or corrected for), and the stability of the scanner head in the direction perpendicular to the scan must be better than 0.1 micron. These requirements were more difficult for us to satisfy, so the first technique was implemented in tests at FFTB.

From the above it is straight forward to list some of the properties of a grid/membrane for a scanner of this type:

1. The size of the grid must be large enough to permit scans of useful length, for example the $1.99 \mathrm{~mm}$ mentioned above. Future scans must be spaced so they do not overlap with the damage pits of previous scans, and typically will be spaced at least 20 microns from them. Because of the possibility that the beam could move relative to the scanner during tuning, we chose a conservative 50 micron spacing between scans. If 100 scans are anticipated, there must be $2-5 \mathrm{~mm}$ of transverse space available on the grid.

2. The substrate must be thin in radiation lengths relative to the metal lines. It must be remembered that all of the beam will always pass through the membrane, whereas only a fraction of it will be intercepted by the metal. Obviously, uniformity of the substrate thickness over dimensions comparable with the line-to-line spacing is equally important. A peak signal ratio between wire and substrate of $2: 1$ is adequate to give useful scans, provided the substrate background is uniform within $10 \%$.

3. The high- $Z$ lines must be spaced to allow for the measurement of large beams as well as small ones. The technique fails when a beam spot overlaps two metal lines at once, The upper limit of the dynamic range of spot size measurement is effectively R.M.S. < $0.25 \times$ (line spacing).

4. The R.M.S. width of the metal line, determined from its profile, should be less than that of the smallest beam spot to be measured. If the line can be measured well, the range can be extended in the usual way by unfolding the line profile from the scan result.

5. Ideally, vertical, horizontal and diagonal lines should be placed on the same membrane to allow determination of $\sigma_{x}, \sigma_{y}$ and tilt of the electron beam.

All of these requirements can be met by modern lithographic techniques. In principle, line widths of $150-200 \mathrm{~nm}$, adequate for measuring spots down to $\sigma=50 \mathrm{~nm}$, can be prepared using, for example, high aspect ratio electron beam lithography. In a first test of the approach, we have manufactured grids of several patterns, using two different substrates. We were able to expose some of these to a beam already measured by a carbon fiber wire scanner at FFTB, use them repeatedly to remeasure the spot size, and eventually use microscopes to examine the damage caused by the beam. 


\section{Sample Preparation}

The samples were prepared lithographically on silicon wafers polished on both sides. An array of $10 \mathrm{~mm}$ square chips was made. In the middle of each square, a $5 \mathrm{~mm}$ square membrane was formed. Two types of membrane were made - of $\mathrm{Si}_{3} \mathrm{~N}_{4}$ and of silicon.

The silicon nitride was grown to a depth of $250 \mathrm{~nm}$ on both sides of the cleaned wafer in a low-stress chemical vapor deposition (CVD) process. On the back side a $5 \mathrm{~mm}$ square window was patterned and the $\mathrm{Si}_{3} \mathrm{~N}_{4}$ was removed from it by plasma etching. On the front, a $1 \mu \mathrm{m} \mathrm{Al}$ layer was deposited and the wafer masked with the line pattern. After development, the wafer was wet-etched to produce an undercut in the aluminum layer. This guaranteed fairly sharp side walls for the deposited metal lines. Subsequently a $10 \mathrm{~nm}$ thick seed layer of $\mathrm{Cr}$ was grown as a foundation for the lines, which were deposited as a $0.3 \mu \mathrm{m}$ Au layer. Some samples with $0.6 \mu \mathrm{m}$ lines of $\mathrm{Cr}$ were also made. Lift off was performed in a weak $\mathrm{KOH}$ solution which also removed the aluminum. Finally the supporting silicon was removed from the window region by isotropically wet-etching from the back, leaving the thin $\mathrm{Si}_{3} \mathrm{~N}_{4}$ membrane. The structure was stable enough to be handled carefully in air.

The process was similar for the silicon membranes, except that the material was doped with boron to form an etch-stop, and etching the back side resulted in a membrane thickness of $2.5 \mu \mathrm{m}$. The gold lines deposited in this case were $0.6 \mu \mathrm{m}$ thick.

Patterns of lines, nominally $2 \mu \mathrm{m}$ wide and spaced at $20 \mu \mathrm{m}$, were formed over some wafers. Others used spacing of $21.25 \mu \mathrm{m}$. In fact the line widths were found to vary between 1.5 and $2.6 \mu \mathrm{m}$, depending on the exposure of the photomask. The silicon "picture frame", 2.5 $\mathrm{mm}$ wide, around the membrane allowed easy handling and mounting. The sample shown in Figure 2 has been used for scanning, and scars from the electron beam can be seen in the translucent silicon membrane area.

\section{Experimental arrangements}

For the tests at FFTB, some sample metal grids were mounted on a rigid rod. The rod also held a conventional fork with $4 \mu \mathrm{m}$ carbon wires in the same plane as the grids (Figure 3). This was installed in vacuum at the FFTB focus. It was attached rigidly, through bellows, to an external $x-y$ stage driven by stepping motors. A thin square corner of aluminum was provided to allow the initial relative position of the beam and scanner to be ascertained in both $\mathrm{x}$ - and $\mathrm{y}$-axes. This was used by manipulating the device until Bremsstrahlung from the beam was detected, first from one edge of the aluminum, and then from the other.

The electron beam was established and tuned, making use of the standard carbon wire scanning system to characterize the beam spot. The procedure involves stepping the beam across a wire by using air-core electromagnets. Obviously it was not possible to measure spots smaller than would destroy the carbon wires. At this point a few hours were allocated to us for tests of the wire grids. During this time it was expected that the beam spot size would slowly drift away from the measured setting.

The grids were moved into the beam and positioned with the beam at a selected starting point. Bremsstrahlung was detected by using the standard FFTB detector package [5]. 
As described above, a scan was performed by moving the grid synchronously with the $10 \mathrm{~Hz}$ beam repetition rate, stopping for each beam pulse. The step sizes were equal to the grid spacing plus an increment equal to the resolution desired for the beam overlap peak.

\section{Experience with $\mathrm{Si}_{3} \mathrm{~N}_{4}$ Membranes}

The silicon nitride membranes were the first to be tested. The electron beam was set up initially with an intensity of $9 \times 10^{9}$ electrons per bunch, and focussed to $\sigma_{x}=4 \mu \mathrm{m}$ and $\sigma_{y}$ $=0.6 \mu \mathrm{m}$. A carbon wire was lost in this process. The first metal wire grid $(0.3 \mu \mathrm{m} \mathrm{Au} \mathrm{lines})$ was then moved into the beam, but broke immediately. Later examination under an optical microscope found that the broken membrane had curled up under its own tension against the four edges of the window. Numerous cracks were visible in the surviving sections.

Later, with the beam intensity reduced to $6 \times 10^{9}$, a second wire grid $(0.6 \mu \mathrm{m} \mathrm{Cr}$ lines) was brought to the beam. This withstood 12 scans before breaking. Figure 4 shows an area of the membrane that was still observable under an optical microscope despite the curling. The trails of damage pits left by the beam are clearly visible. The puncture point was caused by "parking" the beam for several pulses. It is noticeable that the shape of its rosette of cracks is considerably influenced by the presence of the metal lines.

For the first sample, representative sizes of the damage pits were $4.1 \mu \mathrm{m} \times 13.1 \mu \mathrm{m}$, and the beam spot ellipse was evidently tipped by $14^{\circ}$. In this case the substrate was punctured at every beam hit. When the beam hit the middle of a metal line, the area of the puncture was about three times larger, since the energy deposited by the beam was much larger. The damage points on the second foil, which was exposed to the lower intensity beam, did not puncture the membrane except where the beam hit a metal line. Representative damage pit sizes were $3.5 \mu \mathrm{m} \times 7.3 \mu \mathrm{m}$. These damage profiles compare with beam sizes measured to be in the range $0.8<\sigma_{y}<1.5 \mu \mathrm{m}$ and $\sigma_{x}=4 \mu \mathrm{m}$.

Despite the destruction of the grids, several successful beam scans were recorded, with the profiles available in real time. The measurements of vertical spot sizes just mentioned came from these scans. The example in Figure 5 shows a beam profile that, after quadrature subtraction of the R.M.S. width $(0.75 \mu \mathrm{m})$ of the wires, yields a beam width of $\sigma_{y}=0.83 \mu \mathrm{m}$.

\section{Experience with Silicon Membranes}

The stress in the $\mathrm{Si}_{3} \mathrm{~N}_{4}$ substrates, evident in the strong curl after fracture, probably contributed to their loss. The silicon substrates on the silicon frames should be free of these stresses. The greater thickness of these membranes $\left(2.5 \mu \mathrm{m}\right.$ versus $0.25 \mu \mathrm{m}$ for $\left.\mathrm{Si}_{3} \mathrm{~N}_{4}\right)$ also added to their ruggedness. Electrical conductivity may also play a part by removing any tendency for tree-like fracture.

The beam test of these grids was carried out at a later date than that above. Both $\mathrm{Si}_{3} \mathrm{~N}_{4}$ and $\mathrm{Si}$ membranes were available. The initial tuning of the beam left a spot size of $\sigma_{\mathrm{y}}=1.7$ $\mu \mathrm{m} \times \sigma_{\mathrm{x}}=6.4 \mu \mathrm{m}$ at $7 \times 10^{9}$ electrons per pulse. An initial test of a $\mathrm{Si}_{3} \mathrm{~N}_{4}$ sample led to another failure after a few scans. When scanning was carried out on a grid with a silicon 
substrate, however, 24 scans were accumulated without failure. Subsequent microscopic examination showed no sign of the cracking that characterized the $\mathrm{Si}_{3} \mathrm{~N}_{4}$. Three grids with silicon substrates were exposed, for various numbers of scans, without failure.

The scan profiles were again available on-line, and they showed that, as expected, the thick silicon produced a much increased background level. This was, however, uniform enough that the data was easily fitted to a peak with a flat background. An example is shown in Figure 6.

A photomicrograph (Figure 7) shows part of a grid with lines of damage from 24 scans. Dark marks indicate a pit, bright spots show where the beam punctured the membrane. At higher magnification, as in Figure 8, one can see the increase in damage that occurred as the beam increasingly overlapped a metal line.

Towards the end of the tests, the beam profiles obtained from the scans had a shape near the peak that was wider than expected for a Gaussian beam shape, even allowing for the fluctuations in the background rate. In subsequent microscopic examination, damage craters showing a double lobed shape were seen (Figure 9). A beam shape with two slightly different Gaussians $\sim 0.9 \mu \mathrm{m} \times 5 \mu \mathrm{m}$, separated along the minor axis by $2.5 \mu \mathrm{m}$, is reasonably consistent with both sets of observations.

\section{Conclusions}

We have demonstrated the functioning of a compact replacement for linac wire scanners, designed to work in beams intense enough to be destructive. The technique makes use of thin silicon membranes embossed with metal lines, and it can readily be incorporated into an already existing wire scanner control system. However, since it accumulates damage as it is used, the system must use a fresh surface for each scan, and so must retain a map of already-used areas. These grids are inexpensive to make in quantity, and several of them can be placed on the scanner head at once. Hundreds of scans can be made before a vacuum access is needed to replace them.

Grids made with very fine lines may be able to measure spots as small as $\sigma=50 \mathrm{~nm}$. In this case, lines of $200 \mathrm{~nm}$ full width produced by high aspect ratio lithography would suffice. There has not yet been an opportunity to make a test with spots in the $100 \mathrm{~nm}$ range.

\section{Acknowledgements}

We thank Prof. David Burke and Dr. Peter Tenenbaum for help with the set-up of the electron beam. Karl Bouldin and Yung-Yung Sung gave valuable assistance with the assembly and the control hardware. We also thank Marnel King at CIS Stanford for preparation of the $\mathrm{Si}_{3} \mathrm{~N}_{4}$ samples, and Kim Lee of IBM Yorktown Heights for preparation of the $\mathrm{Si}$ membranes. One of us (C.H.B.) acknowledges the Swiss National Fund for financial support. 


\section{References}

[1] R. Fulton et al., Nucl. Instr. and Meth. A 274 (1989) 37.

[2] G. Bowden et al., Nucl. Instr. and Meth. A 278 (1989) 664.

[3] C. Field et al., Nucl. Instr. and Meth. A 295 (1990) 279

[4] M.C. Ross et al., Proc. Accelerator Instrumentation Workshop, Berkeley, 1992, AIP Conf. Proc. 281 (1993) 264.

[5] C. Field, Nucl. Instr. and Meth. A 360 (1995) 467

[6] J. Buon et al., Nucl. Instr. and Meth. A 306 (1991) 93; T. Shintake, Nucl. Instr. and Meth. A 311 (1992) 453; V. Balakin et al., Phys. Rev. Lett. 74 (1995) 2479. 


\section{Figure Captions}

1. Illustration of the steps in a scan: (a) scan performed perpendicular to the metal lines; (b) scan performed using a single line.

2. An example of a metal grid showing the silicon support frame and the translucent silicon membrane. The outer dimensions are $1 \mathrm{~cm}$. square. Lines of damage pits caused by the electron beam can be seen near the center.

3. Drawing of the in vacuo section of the scanning structure. Six grids similar to Figure 2 are supported between aluminum rails. At the bottom is a ceramic fork with thin carbon fibers. In the semicircular gap in the ceramic is the thin aluminum foil rectangle used to locate the beam in $\mathrm{x}$ - and $\mathrm{y}$-axes.

4. Part of a $\mathrm{Si}_{3} \mathrm{~N}_{4}$ sample after scanning. Beam damage pits and severe cracking of the substrate are clearly seen. In this sample, the beam ellipse is seen to be tilted by $13^{\circ}$ relative to the horizontal.

5. Beam profile from a scan using a $\mathrm{Si}_{3} \mathrm{~N}_{4}$ substrate. The fitted width of the peak is $\sigma=$ $1.11 \pm 0.05 \mu \mathrm{m}$, which, after unfolding the width of the metal lines, gives a beam size of $0.82 \pm 0.13 \mu \mathrm{m}$.

6. Beam profile from a scan using a silicon substrate. The poorer signal-to-background ratio is evident. The fitted width is $\sigma=1.21 \pm 0.10 \mu \mathrm{m}$, and after unfolding the line width, the beam size is $0.98 \pm 0.14 \mu \mathrm{m}$.

7. Micrograph of part of a foil showing lines of damage pits from 24 scans.

8. Higher magnification view of part of Figure 2. The roughly elliptical damage pits are increased in size and distorted when the beam encounters a metal line.

9. Scanning electron microscope image of a section of the grid of Figure 8. The damage spots show that the beam had a double-lobed shape. The $2.13 \mu \mathrm{m}$ metal lines are $20 \mu \mathrm{m}$ apart. 


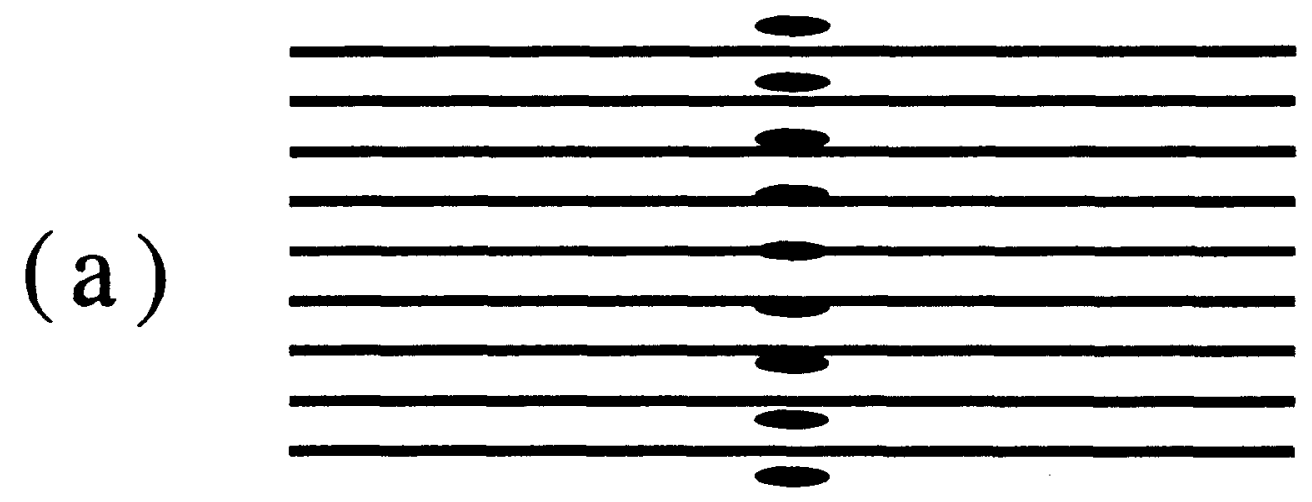

Fig. 1

(a)

Fig. 1

\section{(b)}




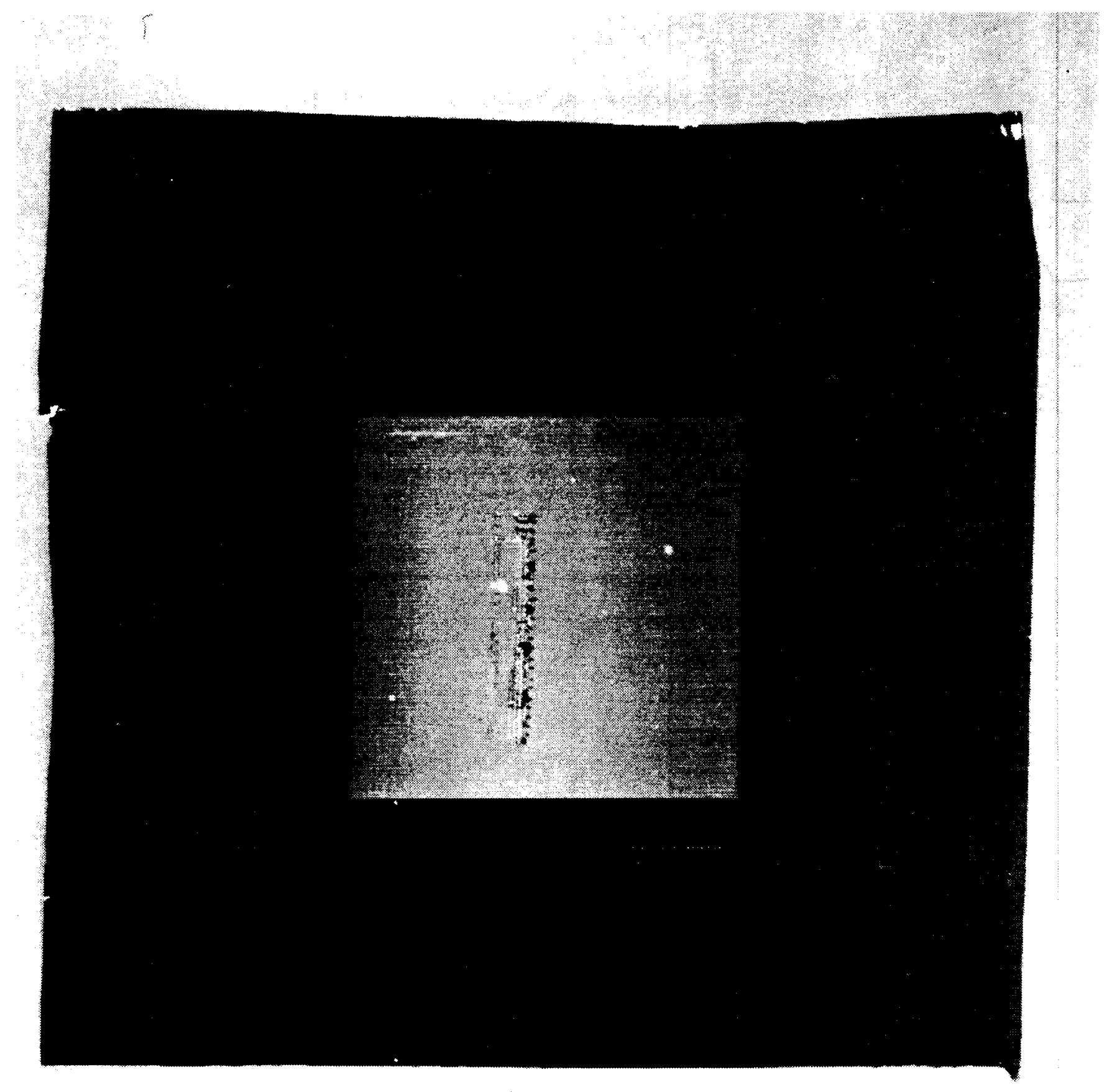

Fig. 2 


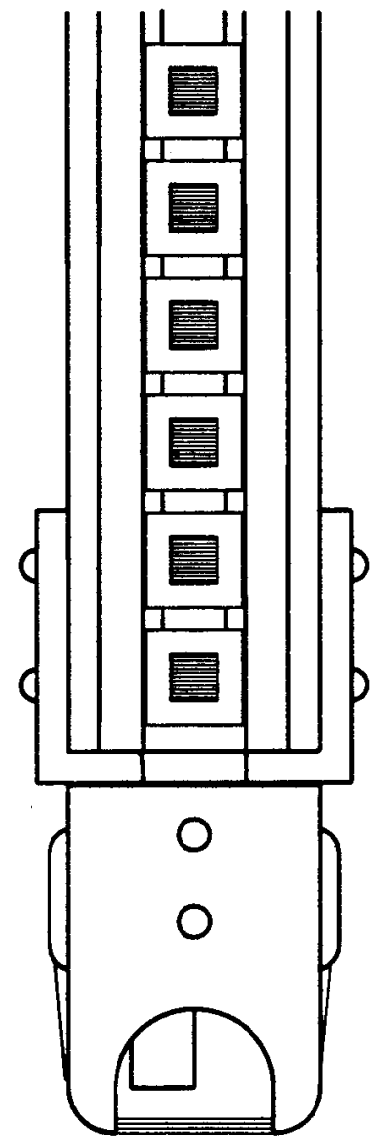

Fig. 3 


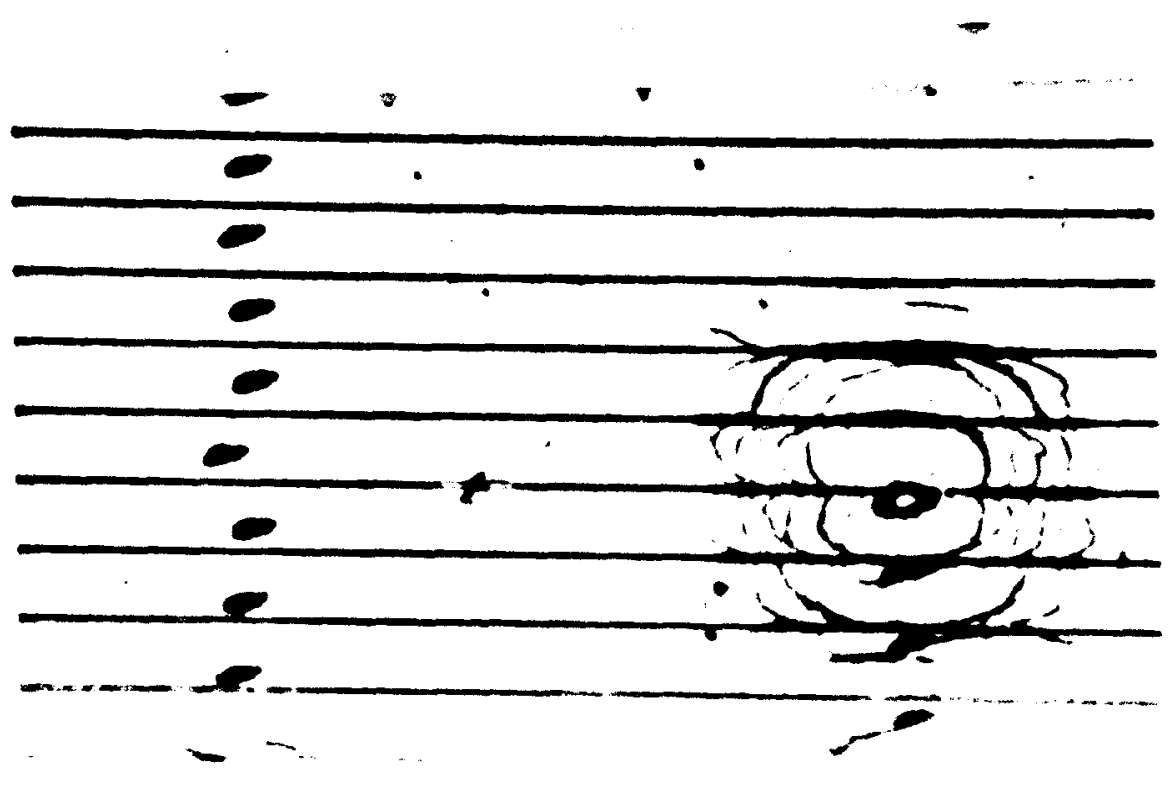

Fig. 4 


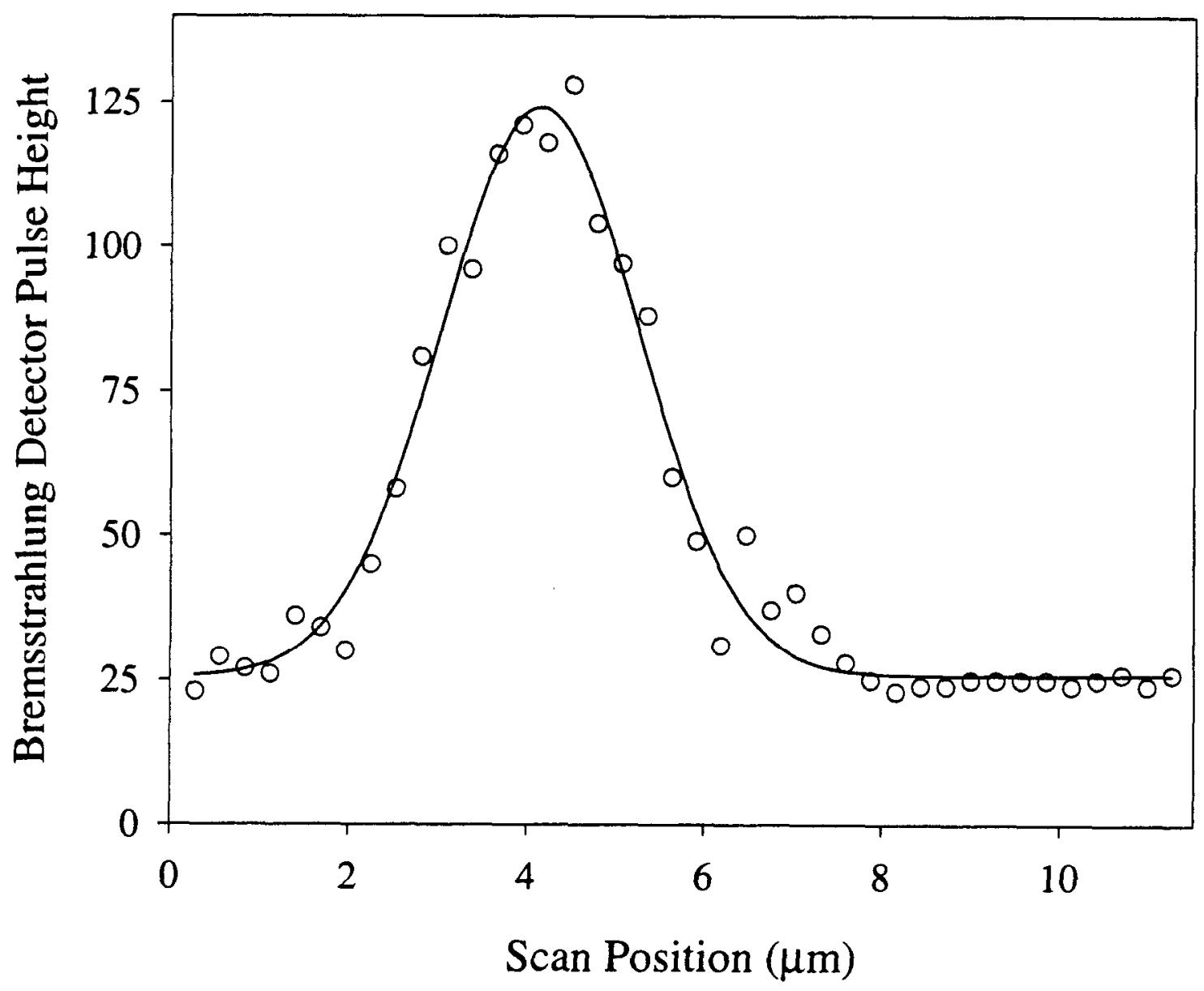

Fig. 5 


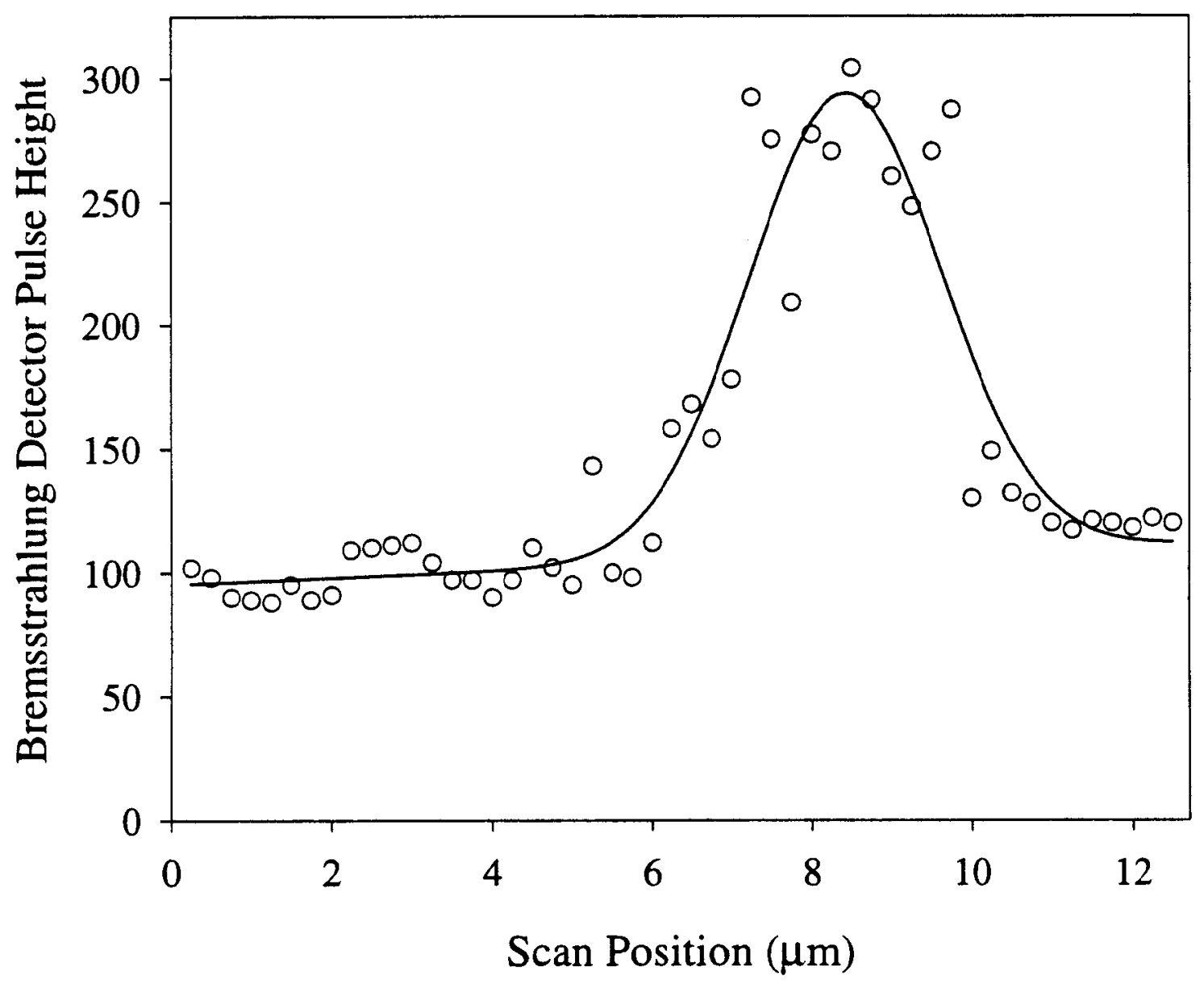

Fig. 6 


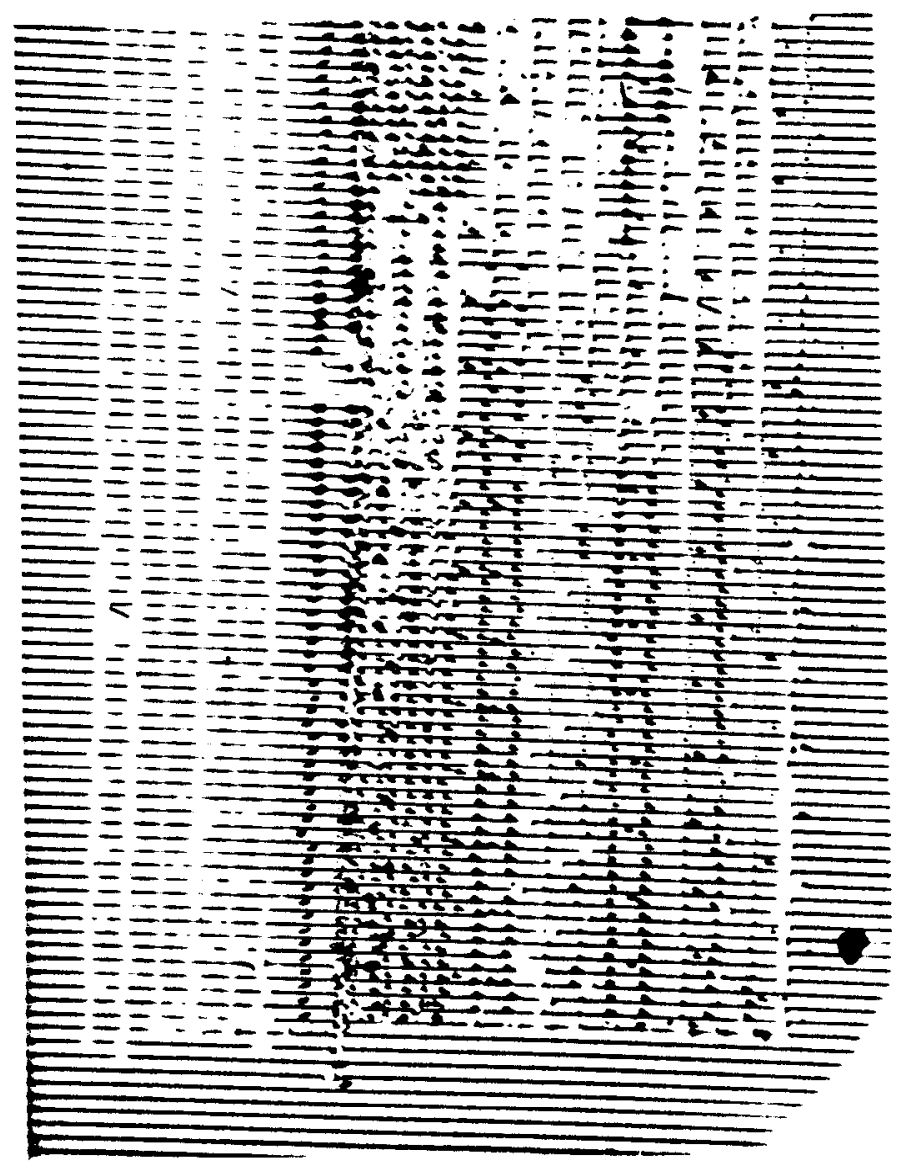

Fig. 7 


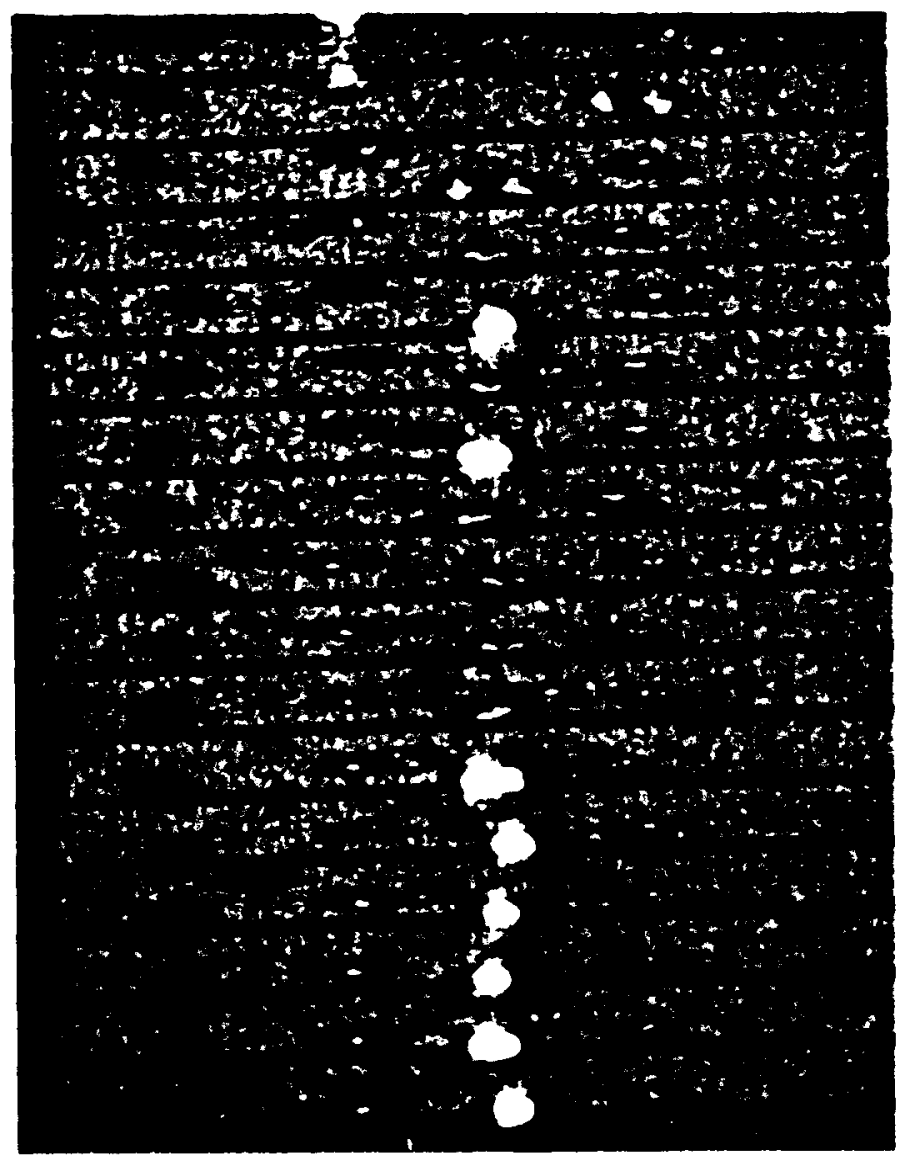

Fig. 8 


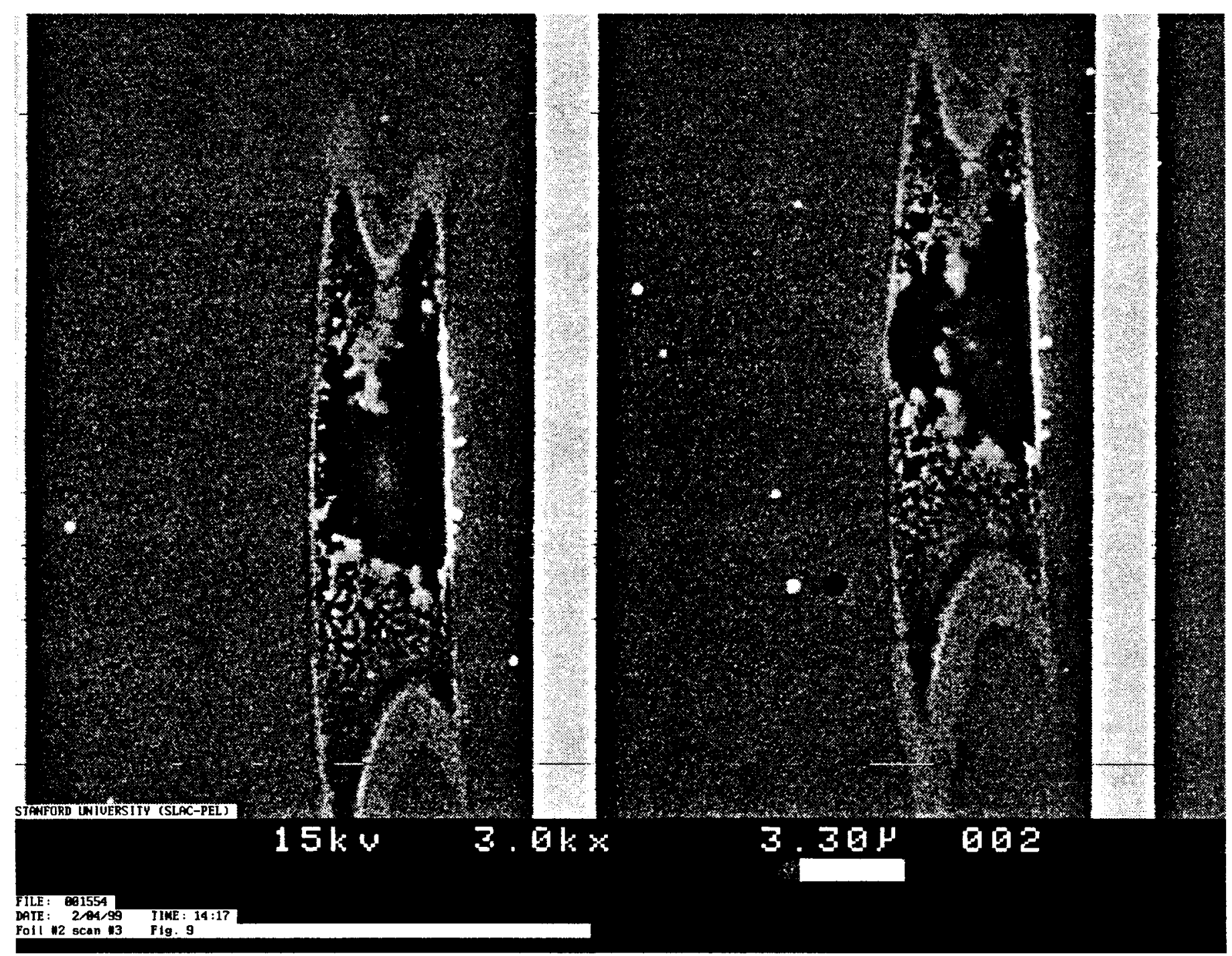

Fig. 9 